\title{
How Humans Control Arm Movements
}

\author{
B. Berret ${ }^{a}$, J.-P. Gauthier ${ }^{b}$, and C. Papaxanthis ${ }^{a}$ \\ Received January 2007
}

\begin{abstract}
This paper is devoted to the behavior of human arms during pointing movements. Several assumptions have already been made about the planning of such motions. None of these assumptions is able, up to now, to explain certain nonintuitive dynamic phenomena, in particular certain asymmetries in the motion and certain time intervals of inactivity of the muscles. In this paper, we propose an assumption explaining all these phenomena. Two strong points in this work are the following. First, our assumption is that human beings minimize a certain criterion that physically makes sense, namely, a compromise between the absolute work of external forces and a comfort term. Second, our conclusions do not rely on any numerical experiment and are completely justified mathematically (i.e., without any argument from simulation or "experimental mathematics," such arguments being usually considered as acceptable in neurobiology). Also, the conclusion that total inactivity holds during some time subintervals of the movement is shown to be a stable property (in our model).
\end{abstract}

DOI: $10.1134 / \mathrm{S} 0081543808020053$

\section{INTRODUCTION}

This work lies in the following very general context: we want to understand motor control for human beings. We want also to understand how humans learn to control the movements of eyes, arms, legs, .... In particular, and in the perspective of long spatial trips, we want to elucidate the role of gravity (or of changes in gravity) in this setting. This study, partly supported by the French "National Center of Spatial Studies" (CNES), concerns one of the most basic problems: the (vertical) pointing (or point-to-point) movements of a human arm.

In the paper [4], there is a recent review about "optimality principles in sensorimotor control," and although it is the only general reference we give here from the neurobiology literature, there is an enormous amount of such literature.

Many of the contributions are like that: choose some criterion (most classical criteria are called "minimum jerk," "minimum torque change," and, in a stochastic context, "minimum variance"). The criterion being chosen, make certain numerical computations to solve the corresponding optimal control problem, and compare to some experiments.

Let us assume all along our discussion that the experiments are in fixed time $T$. This means that a prisoner is required to make a point-to-point movement of the arm, specified by the initial position of the arm, by some target point in the space to be pointed at the end of the motion, and by two sound beeps giving the beginning and the end of the motion. For the sake of simplicity, we consider single-joint movements around the shoulder joint. This means that the prisoner is also required to keep the arm straight. Depending on what we want to study, the prisoner may learn the problem as a first step, or, on the contrary, he may be subject to changes of the target position or of the gravity field.

\footnotetext{
${ }^{a}$ Laboratoire Motricité et Plasticité, INSERM U887, Université de Bourgogne, BP 27877, 21078, Dijon, France.

${ }^{b}$ Laboratoire LE2I, UMR CNRS 5158, Université de Bourgogne, BP 47870, 21078, Dijon, France.

E-mail address: gauthier@u-bourgogne.fr (J.-P. Gauthier).
} 


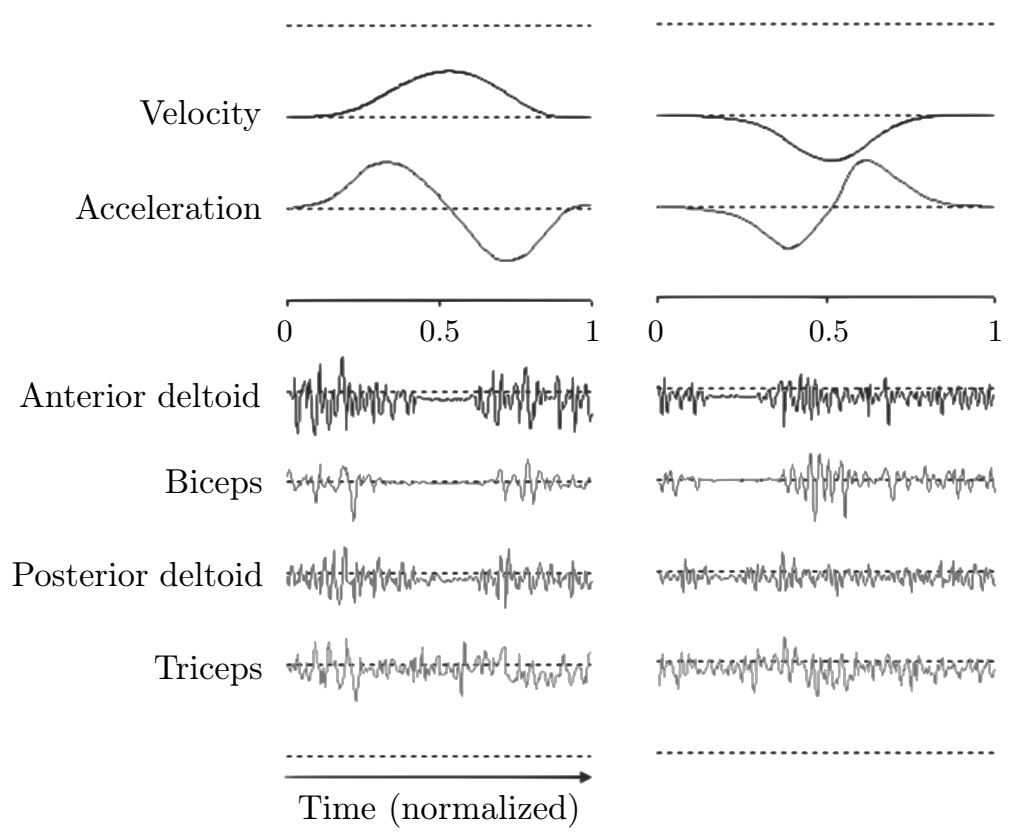

Fig. 1. Experimental data: the time and amplitude of kinematic and electromyographic measurements are normalized.

Just as an example, the minimum jerk problem is the following: minimize the rate of change of the angular acceleration of the arm, i.e.,

$$
\min \int_{0}^{T}\left(\frac{d^{3} \theta}{d t^{3}}(t)\right)^{2} d t .
$$

Immediate computation shows that the optimal velocity profile is given by

$$
\frac{1}{\omega} \dot{\theta}(\tau)=\tau^{2}(1-\tau)^{2}
$$

where $\tau=\frac{t}{T}$ is the normalized time and $\omega$ is a constant depending on the target angle. This is a completely symmetric profile with zero derivatives at the endpoints, the maximum being reached at $\tau_{\mathrm{M}}=0.5$.

In fact, real velocity profiles of vertical arm movements do not fit well with this profile (see $[5,6])$ and with any of those from the literature. Figure 1 shows two experimental profiles. Measurements are made with sensors planted on the muscles of the prisoner (electromyographic signals) and with an accelerometer. The first column is an upward motion, and the second column corresponds to a downward motion.

In both cases, the triceps is always activated: one of the reasons is that the arm needs to keep straight (no motion at the level of the elbow). For downward movements, after the beginning of the motion, there is always some activation (at least of the posterior deltoid). For the upward motion, one checks very clearly inactivation of all muscles (except triceps) during some time interval in the second part of the motion.

Remark 1. This inactivity sequence appears exactly where it is predicted in the paper (a bit after the maximum velocity). However, it does not reflect on the acceleration profile. What is displayed in the picture is just the brute acceleration measured by an accelerometer, which is already smoothed and requires some corrections. Moreover, there could be some residual component due to the activity of the triceps. 
The main qualitative behaviors that are not reproduced by classical models (criteria) are as follows:

1. The asymmetry in the velocity profile (maximum is a bit before the half of the interval) from $0.45 T$ to $0.49 T$, and the difference between upward and downward movements.

2. As we have explained just above, there is some time interval after the middle of the interval on which activation of the muscles is almost zero. This is very clear for movements in the upward direction, although it is not visible for downward movements.

Experiments have been made in normal gravity $(1 g)$ and microgravity $(0 g)$. In the zero gravity case, the velocity profiles are symmetric (as in the case of minimum jerk).

In this paper, we investigate a criterion that seems to explain more or less everything, in a qualitative and quantitative way. We mean that both these unexpected asymmetries and the zero excitation interval are explained, even quantitatively. In the zero gravity case, we still get symmetric motions.

The criterion is the following:

$$
J(u)=J_{1}(u)+J_{2}(u)=\int_{0}^{T}|\dot{\theta}(t) u(t)| d t+\int_{0}^{T} \alpha(\dot{\theta}) \ddot{\theta}(t)^{2} d t,
$$

where $\alpha(y)=\alpha_{\mathrm{U}}$ for $y>0$ and $\alpha(y)=\alpha_{\mathrm{D}}$ for $y<0$, with $\alpha_{\mathrm{D}}>\alpha_{\mathrm{U}}>0$. The variable $u$ is the torque applied by the muscles to the arm (the control variable). Of course, we also assume absolute bounds $-u_{\min } \leq u \leq u_{\max }$ on the control $u$.

The significance of this criterion is the following: it is a compromise between $J_{1}(u)$ and $J_{2}(u)$. The term $J_{1}(u)$ represents the absolute work of the forces developed by the muscles (remember that the infinitesimal work of a torque $u$ is $d w=u d \theta=u \dot{\theta}(t) d t)$. The term $J_{2}(u)$ is a comfort term, expressing that the human articulations do not like high acceleration (anyone can check this very easily). The fact that $\alpha_{\mathrm{D}}>\alpha_{\mathrm{U}}$ is not very important (although it causes certain technical complications here) and could be omitted in the exposition. It means that the articulations are more sensitive to high acceleration for motions in accordance with the gravity than for motions against the gravity. Without this fact $\alpha_{\mathrm{D}} \neq \alpha_{\mathrm{U}}$, certain quantitative differences between upward and downward motions cannot be explained.

For this criterion, contrary to what is done usually in computational neuroscience (numerical experiments), it is not a so hard exercise to solve explicitly the minimization problem for a rigid arm in a gravity field. This is what we do in the paper, using Pontryagin's maximum principle [1]. Analyzing the results, the reader will easily understand that it is certainly unexpectable to get these results just by numerical investigation.

Let us briefly describe the optimal synthesis we get. The most complicated optimal trajectories (in fact, those that occur in practice) are of the type bang-singular-bang (for upward motion: maximum control, singular control, minimum control). Due to the term $|\dot{\theta}(t) u(t)|$ in the energy part $J_{1}$ of the criterion, the singular piece is divided in three pieces: usual singular piece, inactivation interval, and usual singular piece (by "usual singular" we mean a piece along which the Hamiltonian is differentiable with respect to the control $u$, although the "special singular" piece, i.e., the inactivation piece, corresponds to the fact that the maximum of the Hamiltonian is attained at a point of nonsmoothness with respect to $u$ ).

In Fig. 2, we have depicted the results we get for an upward motion (in this figure, we have also taken into account inertial control, which is clearly the case in practice in Fig. 1; see Section 4 for details). It is the reason why we have moreover a gradient constraint reached at the beginning and at the end of the motion. In this picture, one can see very clearly the inactivation interval. For downward motion, it almost disappears. 

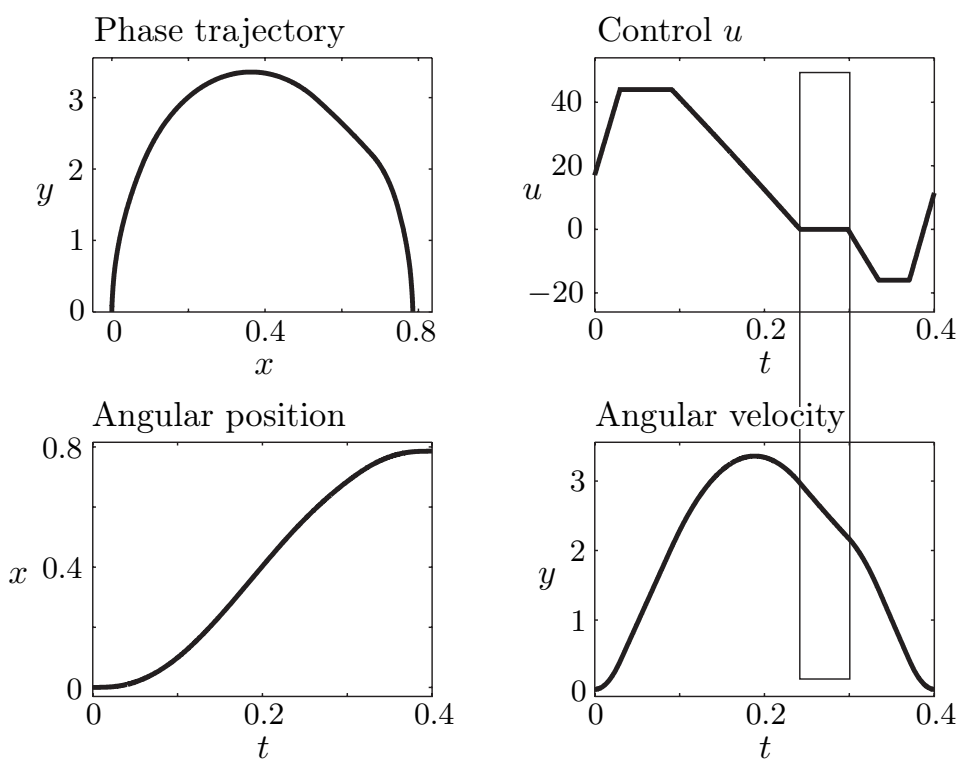

Fig. 2. The results we get for an upward motion. The phase of inactivation of the muscles is shown.

The paper is divided as follows.

In Section 2, we state our basic optimal control problem. We deal with the well-posedness (which is not obvious) and with the existence of solutions.

Section 3 summarizes completely the results of our study, without proof.

In Section 4, we deal with a real smoothed version (inertial control) of our results that seems to be more in accordance with practical observations (we add gradient constraints on the control to get zero derivatives at the endpoints of the velocity profiles). We show that, provided that these constraints are large enough, the only effect is smoothing the strategy at the endpoints of the optimal trajectories; i.e., we get, as expected, zero derivatives at the endpoints of the velocity profiles (which is always observed in practice), but all the other intermediate behaviors remain unchanged.

In Section 5, we go to one of our main conclusions of practical interest (also true without the "small-angle" assumption): the inactivation principle.

In Section 6 we give some hints to get the results of Section 3.

\section{STATEMENT OF THE PROBLEM}

2.1. System under consideration. Consider a one-degree-of-freedom rigid arm moving in a vertical (with respect to the gravitational force) plane, subject to the following equations, in which we neglect the friction terms:

$$
\left\{\begin{array}{l}
\dot{x}=y \\
\dot{y}=u-k \cos x
\end{array}\right.
$$

where

- $x$ is the position of the arm (the angle between the horizontal axis and the arm),

- $y$ is the angular velocity,

- $k$ is a constant (depending on the gravity, the mass of the arm, its inertial moment, and the position of its center of mass).

It is a Hamiltonian system with external force (torque) $u$, the corresponding Hamiltonian being

$$
\mathcal{H}=\frac{y^{2}}{2}+k \sin x-u x
$$


where the term $\frac{y^{2}}{2}$ corresponds to the kinetic energy, the term $k \sin x$ is the potential term, and $u x$ is the action of external forces.

Considering only small angles, we write $\sin x=x+O\left(x^{3}\right)$ and approximate the Hamiltonian by

$$
\mathcal{H}=\frac{y^{2}}{2}+(k-u) x
$$

We get the following standard linear control system:

$$
\left\{\begin{array}{l}
\dot{x}=y \\
\dot{y}=u-k .
\end{array}\right.
$$

2.2. Problem under consideration. Along trajectories of the system that connect a certain "source point" $X_{\mathrm{s}}=\left(x_{\mathrm{s}}, 0\right)$ to a certain "target point" $X_{\mathrm{t}}=\left(x_{\mathrm{t}}, 0\right)$, we want to minimize the following cost $J(u(\cdot))$ in fixed time $T$ :

$$
J(u(\cdot))=\int_{0}^{T}\left(|y u|+\alpha(y) \dot{y}^{2}\right) d t
$$

subject to the constraints

$$
-u_{\min } \leq u \leq u_{\max }
$$

and

$$
\begin{cases}\alpha(y)=\alpha_{\mathrm{U}}>0 & \text { if } y>0 \\ \alpha(y)=\alpha_{\mathrm{D}}>\alpha_{\mathrm{U}} & \text { if } y<0 .\end{cases}
$$

This problem, denoted by $(\mathcal{P})$, was discussed in Section 1 .

The optimal cost is denoted by $J^{*}$, and the corresponding minimizers (if any) will be denoted by $u^{*}$ and $X^{*}$, with $X^{*}=\left(x^{*}, y^{*}\right)$.

Remark 2. In problem $(\mathcal{P})$, neither the dynamics, nor the constraints, nor the cost depend on $x$. Hence the solutions depend only on the difference $\Delta_{x}=x_{\mathrm{t}}-x_{\mathrm{s}}$. Therefore, $J$ will be denoted by $J_{T, \Delta_{x}}$ wherever it will be necessary to consider this dependence.

2.3. Well-posedness of the problem. A priori our problem is an ill-posed problem since the value $\alpha(y)$ for $y=0$ is not given.

Theorem 1. (A) Problem $(\mathcal{P})$ is well posed; i.e., the values $J(u(\cdot))$ do not depend on the value $\alpha(0)$.

(B) $J_{T, \Delta_{x}}^{*}$ is a decreasing function of $T$.

(C) Given a trajectory $(u(\cdot), X(\cdot))$, with $X(\cdot)=(x(\cdot), y(\cdot))$, we may consider that there is $0 \leq \widetilde{T} \leq T$ with

(C1) $y=0$ and $u=k$ for $T \geq t \geq \widetilde{T}$

(C2) on the interval $[0, \widetilde{T}], y=0$ on a subset of null Lebesgue measure only.

Proof. For $(\mathrm{A})$, let $E=\{t \in[0, T]$ such that $y(t)=0\}$. Let $\widetilde{E} \subset E$ be the set of times that are not accumulation points of $E$. The set $\widetilde{E}$ is countable. Let $\bar{E}=E \backslash \widetilde{E}$. We have

$$
\int_{\bar{E}}\left(|y u|+\alpha(y) \dot{y}^{2}\right) d t=\int_{\bar{E}}\left(|y u|+\alpha(0) \dot{y}^{2}\right) d t=0,
$$


because $\dot{y}$ exists on a subset of total measure in $\bar{E}$ since $y$ is absolutely continuous, and, at such a point $t$ where $\dot{y}$ exists, $\dot{y}=0$ also (arbitrarily close to $t$, there is another time $t^{\prime}$ with $y\left(t^{\prime}\right)=0$ ). This shows that $J(u(\cdot))$ does not depend on $\alpha(0)$.

Let us assume the existence of minimizers, which will be shown in the next Subsection 2.4. Point (B) is obvious: assume we have found $T_{2}>T_{1}$ with $J_{T_{2}, \Delta_{x}}^{*}>J_{T_{1}, \Delta_{x}}^{*}$, and then consider the control $u_{3}$ defined as follows: $u_{3}$ is $u_{1}$ from time 0 up to time $T_{1}$ and is $k$ from $T_{1}$ on. Clearly, $J_{T_{2}}\left(u_{3}\right)=J_{T_{1}, \Delta_{x}}^{*}<J_{T_{2}, \Delta_{x}}^{*}$. A contradiction.

To prove (C), we start from any trajectory $(u(\cdot), X(\cdot))$ connecting $X_{\mathrm{s}}$ to $X_{\mathrm{t}}$ in time $T$. We will construct another trajectory $(\widetilde{u}(\cdot), \tilde{X}(\cdot))$ connecting the same endpoints and reaching $X_{\mathrm{t}}$ at time $\widetilde{T}$, such that the cost is the same and $y$ is zero only on a subset with zero measure of $[0, \widetilde{T}]$. Let us define the function $\varphi$ by $\varphi(\theta)=$ measure $\{t ; 0 \leq t \leq \theta, y(t) \neq 0\}$. Set $\widetilde{T}=\varphi(T)$. The function $\varphi$ is well defined and increases on the interval $[0, \widetilde{T}]$. We leave to the reader to check that $\varphi$ is measurable. We define $\widetilde{u}$ up to some irrelevant subset of measure zero of the interval $[0, \widetilde{T}]$ by $\widetilde{u}(\varphi(\theta))=u(\theta)$. By construction, this control produces the same trajectory $\widetilde{X}(\cdot)$ as $X$ up to some measurable reparametrization.

2.4. Existence of minimizers. It is not so easy to show that the minimum is reached in this problem. We follow more or less the scheme of a proof in [2], with some additional problems due to the nonsmoothness and even noncontinuity of our cost.

Theorem 2. There is a certain critical time $T_{\mathrm{c}}=\sqrt{\frac{2 \Delta_{x}\left(u_{\min }+u_{\max }\right)}{\left(u_{\min }+k\right)\left(u_{\max }-k\right)}}$ such that, for problem $(\mathcal{P})$, minimizers exist for all $T \geq T_{\mathrm{c}}$.

Proof. First, it is easily seen that for $T<T_{\mathrm{c}}$ there is no admissible trajectory at all connecting the source to the target. Second, for $T=T_{\mathrm{c}}$ there is a single trajectory (described later in Subsection 3.2) connecting the source to the target. Therefore, this trajectory is necessarily optimal. Hence, in this proof we assume $T>T_{\mathrm{c}}$ and claim that the set of admissible trajectories doing the job is nonempty. We will show the existence of some optimal one.

Notation. Changing $u-k$ to $u$, we denote our linear control system by $\dot{X}=A X+B u$. As usual in this machinery, we add an extra variable $z(t)=J_{t}(u)$. The extended control system is written as $\dot{\xi}=G(\xi, u), \xi=(z, X)$. Notice that $G$ is nonsmooth, and even noncontinuous. We also use the convexification of the extended system. By a classical result of Carathéodory, it is the following system:

$$
\begin{aligned}
& \dot{z}=\omega \lambda \varphi\left(u_{1}\right)+\omega(1-\lambda) \varphi\left(u_{2}\right)+(1-\omega) \varphi\left(u_{3}\right), \\
& \dot{x}=y, \\
& \dot{y}=\omega \lambda u_{1}+\omega(1-\lambda) u_{2}+(1-\omega) u_{3},
\end{aligned}
$$

where the controls are $U=\left(u_{1}, u_{2}, u_{3}, \omega, \lambda\right)$ such that $-u_{\min } \leq u_{1}, u_{2}, u_{3} \leq u_{\max }$ and $0 \leq \omega, \lambda \leq 1$. The function $\varphi$ is defined by $\varphi=|y(u+k)|+\alpha(y) u^{2}$.

We write this convexified system in abbreviated notation:

$$
\left(\Sigma_{\mathrm{c}}\right) \quad \dot{\xi}=F(\xi, U),
$$

where $U \in \mathcal{U}$.

Let $U_{k}$ be any minimizing sequence for $\left(\Sigma_{\mathrm{c}}\right)$ and $\xi_{k}$ be the corresponding state sequence. Clearly $\xi_{k}$ is equicontinuous, and by Ascoli $\xi_{k}$ converges uniformly to $\xi^{*}$. We want to show that $\xi^{*}(t)$ is actually a trajectory of $\left(\Sigma_{\mathrm{c}}\right)$ corresponding to some control $U^{*}$.

In fact, $\dot{\xi}_{k}$ converges $*$-weakly to some $\Phi^{*}(t)$. It is enough to show that $\Phi^{*}(t) \in F\left(\xi^{*}(t), \mathcal{U}\right)$.

Let $\mathcal{O}$ be the open set $\mathcal{O} \subset[0, T]$ where $y^{*}(t) \neq 0$. 
First, $\mathcal{O}$ is nonempty: for the subsystem in $X=(x, y)$, which is just the linear system $(\dot{x}=y$, $\dot{y}=v$ ), by standard arguments (see [3] for instance), we have the continuity of the map

$$
v \rightarrow X, \quad L_{[O, T]}^{\infty}, * \text {-weak } \rightarrow C_{[0, T]}^{0}, \text { uniform. }
$$

Therefore, if $\mathcal{O}=\varnothing$, whatever a large $k$, the vector $X_{k}(T)$ cannot approach the target point $X_{\mathrm{t}}$.

Second, for almost all $t \in \mathcal{O}, \Phi^{*}(t)$ is equal to a certain $F\left(\xi^{*}(t), U^{*}\right)$.

Were it otherwise, we could find some subset $\mathcal{S} \subset \mathcal{O}$ of strictly positive measure such that $\Phi^{*}(t) \notin F\left(\xi^{*}(t), \mathcal{U}\right)$ for all $t \in \mathcal{S}$. We can assume that $\mathcal{S}$ is contained in some interval $I \subset[0, T]$ on which $y^{*}(t)$ has constant sign $\left(y^{*}(t)>0\right.$, say). Let us say that the measure of $\mathcal{S}$ is $\varepsilon$ and the measure of $I$ is $l>0$. Then, we can find (by Lusin's criterion) a continuous function $\widetilde{\Phi}(t)$ on $I$ that is different from $\Phi^{*}(t)$ on a set of measure less than $\frac{\varepsilon}{2}$. It follows that there is a subset $\overline{\mathcal{S}}$ (of strictly positive measure) of $I$ such that $\Phi^{*}(t)=\widetilde{\Phi}(t) \notin F\left(\xi^{*}(t), \mathcal{U}\right)$ for all $t \in \overline{\mathcal{S}}$. To finish, there is some $t_{0} \in \mathcal{S}$ such that, for all $\delta$ small enough, the set of $t \in I_{\delta}=\left[t_{0}-\delta, t_{0}+\delta\right]$ such that $\Phi^{*}(t)=\widetilde{\Phi}(t) \notin F\left(\xi^{*}(t), \mathcal{U}\right)$ is denoted by $\mathcal{S}_{\delta} \subset I_{\delta}$, and the measure of $\mathcal{S}_{\delta}$ is strictly positive.

Hence, by the separation of convex sets, there is a $P \in\left(\mathbb{R}^{n}\right)^{*}$ such that $P \Phi^{*}\left(t_{0}\right)=P \widetilde{\Phi}\left(t_{0}\right)>$ $P F\left(\xi^{*}\left(t_{0}\right), \mathcal{U}\right)$. Since $y^{*}\left(t_{0}\right) \neq 0$, since $F(\cdot, \cdot)$ is continuous (recall that $y^{*}>0$ ), and since $\widetilde{\Phi}$ is continuous, it follows that $\delta$ can be chosen small enough for $P \widetilde{\Phi}(t)>P F\left(\xi^{*}(t), \mathcal{U}\right)$ for all $t \in I_{\delta}$. In particular, $P \Phi^{*}(t)>P F\left(\xi^{*}(t), U_{k}(t)\right)$ for all $t \in \mathcal{S}_{\delta}$. This last property is denoted by $(\mathcal{A})$. Also, since $\xi_{k}$ converges uniformly to $\xi^{*}$,

$$
\limsup _{k \rightarrow+\infty} P\left(\Phi^{*}(t)-F\left(\xi_{k}(t), U_{k}(t)\right)\right)>0
$$

for all $t \in I_{\delta}$.

Denoting by $\varkappa$ the indicatrix function of $\mathcal{S}_{\delta}$ in $[0, T]$, we have, by the $*$-weak convergence of $\dot{\xi}_{k}$ to $\xi^{*}$,

$$
\limsup _{k \rightarrow+\infty} P \int_{0}^{T} \varkappa(t)\left(\Phi^{*}(t)-F\left(\xi_{k}(t), U_{k}(t)\right)\right) d t=0 .
$$

This contradicts $(\mathcal{B})$.

We conclude that $\xi^{*}$ is an admissible trajectory of the convexification $\left(\Sigma_{\mathrm{c}}\right)$ : $\Phi^{*}(t)=F\left(\xi^{*}(t), U^{*}(t)\right)$ for almost all $t \in[0, T]$, for some measurable $U^{*}(\cdot), U^{*}(t) \in \mathcal{U}$ (the measurability of $U^{*}$ is a standard exercise).

Third, let us show that $(\mathcal{C}) \xi^{*}$ is in fact an admissible trajectory of the original (nonconvexified) extended system.

Let us consider any fixed $t_{0} \in[0, T]$ such that $y^{*}\left(t_{0}\right) \neq 0$. Then, on some neighborhood of $t_{0}$, our trajectory (of the convexified system $\left(\Sigma_{\mathrm{c}}\right)$ ) has to meet the maximum principle. Hence, the Hamiltonian $H(t)=P F\left(\xi^{*}(t), U^{*}(t)\right)$ has to be maximum with respect to $U, H(t)=p \dot{z}+q y+r \dot{y}$. Moreover, $p(t)=p\left(t_{0}\right)=p$. If $p=0$ (singular case), this obviously leads to

$$
u=\omega \lambda u_{1}+\omega(1-\lambda) u_{2}+(1-\omega) u_{3}=-u_{\min } \text { or } u_{\max },
$$

which obviously implies $u_{1}=u_{2}=u_{3}=-u_{\min }$ or $u_{\max }$, and $\dot{z}=\varphi(y, u)$; therefore, $\xi^{*}$ is a trajectory of the original extended system.

Let us now examine the case where $p \neq 0$. The modified Hamiltonian $\widetilde{H}=p \dot{z}+r \dot{y}$ is maximum with respect to $U$. But

$$
\begin{aligned}
\widetilde{H} & =p\left(\omega \lambda \varphi\left(u_{1}\right)+\omega(1-\lambda) \varphi\left(u_{2}\right)+(1-\omega) \varphi\left(u_{3}\right)\right)+r\left(\omega \lambda u_{1}+\omega(1-\lambda) u_{2}+(1-\omega) u_{3}\right) \\
& =\omega \lambda\left(p \varphi\left(u_{1}\right)+r u_{1}\right)+\omega(1-\lambda)\left(p \varphi\left(u_{2}\right)+r u_{2}\right)+(1-\omega)\left(p \varphi\left(u_{3}\right)+r u_{3}\right) .
\end{aligned}
$$


We shall only examine the case $\omega \lambda \neq 0$. The other cases $(\omega(1-\lambda) \neq 0$ or $(1-\omega) \neq 0)$ are similar. The function $p \varphi(u)+r u$ is strictly concave since $p<0$. But $p \varphi\left(u_{1}\right)+r u_{1}$ has to be maximum, and the maximum is reached at a unique $u^{*}$ (depending on $y$ only). At the end $\widetilde{H}=p \varphi\left(u^{*}\right)+r u^{*}$, $\dot{y}=u^{*}$, and $\dot{z}=\varphi\left(u^{*}\right)$. This shows that the piece of the trajectory $\left(\xi^{*}(t), U^{*}(t)\right)$ around $t_{0}$ is in fact a trajectory of the nonconvexified extended system.

At almost all points $t_{0}$ where $y^{*}\left(t_{0}\right)=0$ (that is, at nonisolated points of $\mathcal{O}$ where $\xi^{*}\left(t_{0}\right)$ is differentiable), $\dot{y}^{*}\left(t_{0}\right)=0$ also, and $u\left(t_{0}\right)=\left(\omega \lambda u_{1}+\omega(1-\lambda) u_{2}+(1-\omega) u_{3}\right)\left(t_{0}\right)=0$. By Theorem 1 , we can assume $\alpha(0)=0$. This implies that $\dot{z}\left(t_{0}\right)=0, \dot{x}\left(t_{0}\right)=0, \dot{y}\left(t_{0}\right)=u^{*}\left(t_{0}\right)=0$, and at the end, at almost all $t, \dot{\xi}^{*}(t)=G\left(\xi^{*}(t), u^{*}(t)\right)$, which means that $\xi^{*}(t)$ is a trajectory of our nonconvexified extended system.

\section{SUMMARY OF THE RESULTS}

3.1. Different classes of optimal trajectories. We classify different types of optimal trajectories in terms of the duration $T$ of the motion. When $T$ increases from the critical time $T_{\mathrm{c}}$ towards infinity, we get the successive strategies. The critical time $T_{\mathrm{c}}$ corresponds to the minimum time necessary to connect the source to the target.

Also, it is a consequence of this study that the optimal strategies remain in one of the half-planes $H_{1}$ or $H_{2}$ corresponding to $y \geq 0$ and $y \leq 0$, respectively. Moreover, the optimal trajectories consist of a single piece connecting $\{y=0\}$ to $\{y=0\}$. These optimal arcs are described here for the upper half-plane $H_{1}$ only, the case of $H_{2}$ leading to completely symmetric formulas.

Remark 3. Up to the change of variables $\widetilde{x}(t)=x(T-t), \widetilde{y}(t)=-y(T-t)$, and $\widetilde{u}(t)=$ $u(T-t)$, the problems that consist of moving from $x_{\mathrm{s}}$ to $x_{\mathrm{t}}$ (e.g., upward) and from $x_{\mathrm{t}}$ to $x_{\mathrm{s}}$ (e.g., downward) are equivalent, and consequently the optimal controls $u$ lead to the optimal controls $\widetilde{u}$ (for the fixed value $\alpha_{\mathrm{D}}$ of the parameter $\alpha$ ). Therefore, up to this exchange of $\alpha_{\mathrm{D}}$ and $\alpha_{\mathrm{U}}$, the solutions for upward and downward motions are formally exactly the same. The quantitative difference between downward and upward motions is only determined by the practical value of $\alpha$. However, it is a crucial fact that $\alpha_{\mathrm{D}} \neq \alpha_{\mathrm{U}}$ for the quantitative results.

In the following, $(p, q)$ will denote the adjoint vector of $(x, y)$. Hence, $\left(p_{0}, q_{0}\right)$ is the initial value of the adjoint vector.

We obtain the following seven different optimal strategies that are presented in more detail in Subsection 3.2 and the equations of which are established from the Pontryagin maximum principle $(\mathrm{PMP})$ in Section 6. Each of them is an optimal solution of problem $(\mathcal{P})$, depending on the explicit values of the parameters.

The seven qualitative types of optimal strategies are denoted by $S_{j}, j=1, \ldots, 7$, and correspond to the following sequences of controls:

- $S_{1}$ (bang-max, bang-min):

$$
\left(u=u_{\max }\right) \rightarrow\left(u=-u_{\min }\right)
$$

- $S_{2}$, the most general strategy (bang, singular, special-singular, singular, bang):

$$
\left(u=u_{\max }\right) \rightarrow\left(u=\frac{q-y}{2 \alpha}+k\right) \rightarrow(u=0) \rightarrow\left(u=\frac{q+y}{2 \alpha}+k\right) \rightarrow\left(u=-u_{\min }\right) ;
$$

- $S_{3}$ (singular, special-singular, singular, bang):

$$
\left(u=\frac{q-y}{2 \alpha}+k\right) \rightarrow(u=0) \rightarrow\left(u=\frac{q+y}{2 \alpha}+k\right) \rightarrow\left(u=-u_{\min }\right) ;
$$

- $S_{4}$ (bang, singular, special-singular, singular):

$$
\left(u=u_{\max }\right) \rightarrow\left(u=\frac{q-y}{2 \alpha}+k\right) \rightarrow(u=0) \rightarrow\left(u=\frac{q+y}{2 \alpha}+k\right) ;
$$


- $S_{5}$ (singular, special-singular, singular):

$$
\left(u=\frac{q-y}{2 \alpha}+k\right) \rightarrow(u=0) \rightarrow\left(u=\frac{q+y}{2 \alpha}+k\right) ;
$$

- $S_{6}$ (bang, singular):

$$
\left(u=u_{\max }\right) \rightarrow\left(u=\frac{q-y}{2 \alpha}+k\right)
$$

- $S_{7}$ (singular only):

$$
\left(u=\frac{q-y}{2 \alpha}+k\right) .
$$

The special-singular pieces corresponding to inactivity sequences $u=0$ are due to the term $|u|$ in the criterion, as we said.

In the following subsections we describe in detail these different strategies.

Notation. In the following subsections, we will use the notations $u_{i}(t), q_{i}(t), x_{i}(t)$, and $y_{i}(t)$ for $t \in\left[0, \tau_{i}\right]$ and $i \geq 1$ for the functions $u, q, x$, and $y$ on the interval $\left[\sum_{j=0}^{i-1} \tau_{j}, \sum_{j=0}^{i} \tau_{j}\right]$ with $\tau_{0}=0$. For instance, $u_{2}(t)$ means $u\left(t+\tau_{1}\right)$ for $t \in\left[0, \tau_{2}\right]$ and $u_{3}(t)$ means $u\left(t+\tau_{1}+\tau_{2}\right)$ for $t \in\left[0, \tau_{3}\right]$.

3.2. Case $S_{1}$. Fastest possible movements, critical time $T_{\mathrm{c}}$. We consider first the abnormal $^{1}$ case, corresponding to the quickest possible movement. This solution depends only upon the constraints $u_{\max }$ and $u_{\min }$.

The corresponding equations for the solutions are the following:

- for $t \in\left[0, \tau_{1}\right]$,

$$
\left\{\begin{array}{l}
u_{1}=u_{\max } \\
y_{1}=\left(u_{\max }-k\right) t \\
q_{1}=q_{0}-p_{0} t \\
x_{1}=x_{\mathrm{s}}+\left(u_{\max }-k\right) \frac{t^{2}}{2}
\end{array}\right.
$$

- for $t \in\left[0, T_{\mathrm{c}}-\tau_{1}\right]\left(\tau_{2}=T_{\mathrm{c}}-\tau_{1}\right)$,

$$
\left\{\begin{array}{l}
u_{2}=-u_{\text {min }}, \\
y_{2}=y_{1}\left(\tau_{1}\right)-\left(u_{\min }+k\right) t, \\
q_{2}=q_{1}\left(\tau_{1}\right)-p_{0} t \\
x_{2}=x_{1}\left(\tau_{1}\right)+y_{1}\left(\tau_{1}\right) t-\left(u_{\min }+k\right) \frac{t^{2}}{2},
\end{array}\right.
$$

with

$$
T_{\mathrm{c}}=\sqrt{\frac{2 \Delta_{x}\left(u_{\min }+u_{\max }\right)}{\left(u_{\min }+k\right)\left(u_{\max }-k\right)}}
$$

and the commutation time $\tau_{1}$,

$$
\tau_{1}=\frac{\left(u_{\min }+k\right) T_{\mathrm{c}}}{2 u_{\max }-k+u_{\min }} .
$$

\footnotetext{
$1_{\text {The trajectories corresponding to }} \lambda=0$ ( $\lambda$ is the adjoint vector to the cost) are called abnormal. Note that they are candidates for optimality whatever the cost. Recall that in the paper we also consider regular (or bang) and singular trajectories (the control is not bang). Singular trajectories are divided into usual singular and special singular ones.
} 
3.3. Case $S_{2}$. The practical strategy, five-piece trajectories. This case is the usual one in practice, and it is also the most complicated. When $T \in\left[T_{\mathrm{c}}, T_{1}\right]$, the solutions are the following:

- for $t \in\left[0, \tau_{1}\right]$,

$$
\left\{\begin{array}{l}
u_{1}=u_{\max } \\
y_{1}=\left(u_{\max }-k\right) t \\
q_{1}=q_{0}+\left(u_{\max }-p_{0}\right) t \\
x_{1}=x_{\mathrm{s}}+\left(u_{\max }-k\right) \frac{t^{2}}{2}
\end{array}\right.
$$

- for $t \in\left[0, \tau_{2}\right]$,

$$
\left\{\begin{array}{l}
u_{2}=u_{\max }+\frac{k-p_{0}}{2 \alpha} t \\
y_{2}=y_{1}\left(\tau_{1}\right)+\left(u_{\max }-k\right) t+\frac{k-p_{0}}{4 \alpha} t^{2} \\
q_{2}=q_{1}\left(\tau_{1}\right)+\left(u_{\max }-p_{0}\right) t+\frac{k-p_{0}}{4 \alpha} t^{2} \\
x_{2}=x_{1}\left(\tau_{1}\right)+y_{1}\left(\tau_{1}\right) t+\frac{u_{\max }-k}{2} t^{2}+\frac{k-p_{0}}{12 \alpha} t^{3}
\end{array}\right.
$$

- for $t \in\left[0, \tau_{3}\right]$,

$$
\left\{\begin{array}{l}
u_{3}=0 \\
y_{3}=y_{2}\left(\tau_{2}\right)-k t \\
q_{3}=q_{2}\left(\tau_{2}\right)-p_{0} t \\
x_{3}=x_{2}\left(\tau_{2}\right)+y_{2}\left(\tau_{2}\right) t-\frac{k t^{2}}{2}
\end{array}\right.
$$

- for $t \in\left[0, \tau_{4}\right]$,

$$
\left\{\begin{array}{l}
u_{4}=-\frac{k-p_{0}}{2 \alpha} t \\
y_{4}=y_{3}\left(\tau_{3}\right)-k t-\frac{k+p_{0}}{4 \alpha} t^{2} \\
q_{4}=q_{3}\left(\tau_{3}\right)-p_{0} t-\frac{k+p_{0}}{4 \alpha} t^{2} \\
x_{4}=x_{3}\left(\tau_{3}\right)+y_{3}\left(\tau_{3}\right) t-\frac{k}{2} t^{2}-\frac{k+p_{0}}{12 \alpha} t^{3}
\end{array}\right.
$$

- for $t \in\left[0, \tau_{5}\right]$,

$$
\left\{\begin{array}{l}
u_{5}=-u_{\min }, \\
y_{5}=y_{4}\left(\tau_{4}\right)-\left(u_{\min }+k\right) t, \\
q_{5}=q_{4}\left(\tau_{4}\right)+\left(u_{\min }-p_{0}\right) t, \\
x_{5}=x_{4}\left(\tau_{4}\right)+y_{4}\left(\tau_{4}\right) t-\left(u_{\min }+k\right) \frac{t^{2}}{2} .
\end{array}\right.
$$

The commutation times $\tau_{i}$ are equal to

$$
\begin{gathered}
\tau_{1}=\frac{q_{0}+2 \alpha\left(k-u_{\max }\right)}{p_{0}-k}, \quad \tau_{2}=\frac{2 \alpha u_{\max }}{p_{0}-k}, \quad \tau_{3}=2 \frac{\left(2 \alpha k+q_{0}\right)\left(u_{\max }-k\right)-\alpha u_{\max }^{2}}{\left(p_{0}-k\right)\left(p_{0}+k\right)}, \\
\tau_{4}=\frac{2 \alpha u_{\min }}{k+p 0}, \quad \tau_{5}=\frac{\left(q_{0}+2 \alpha k\right)\left(u_{\max }-k\right)-\alpha\left(u_{\max }^{2}+u_{\min }^{2}+2 k u_{\min }\right)}{\left(p_{0}+k\right)\left(k+u_{\min }\right)} .
\end{gathered}
$$



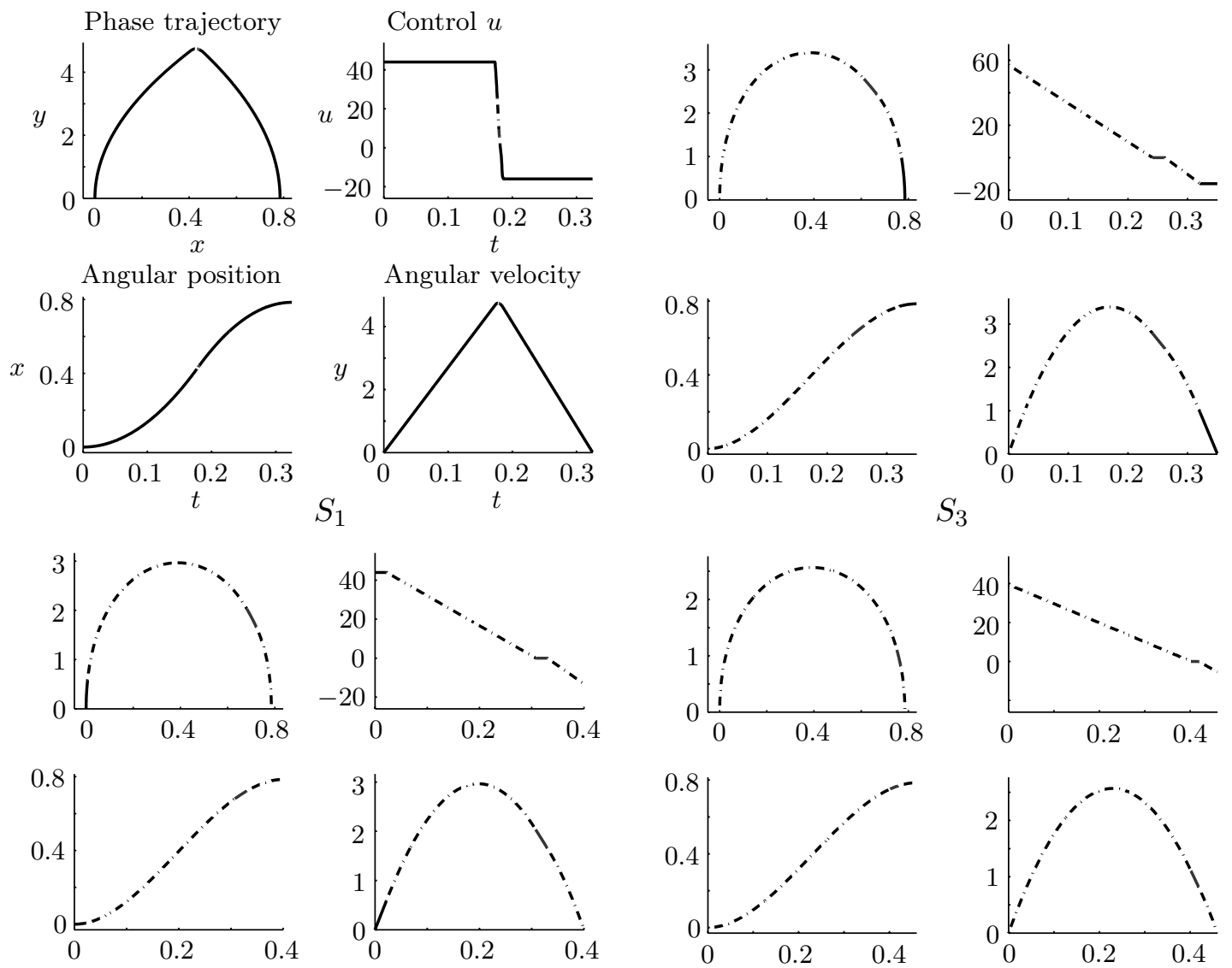

$S_{4}$
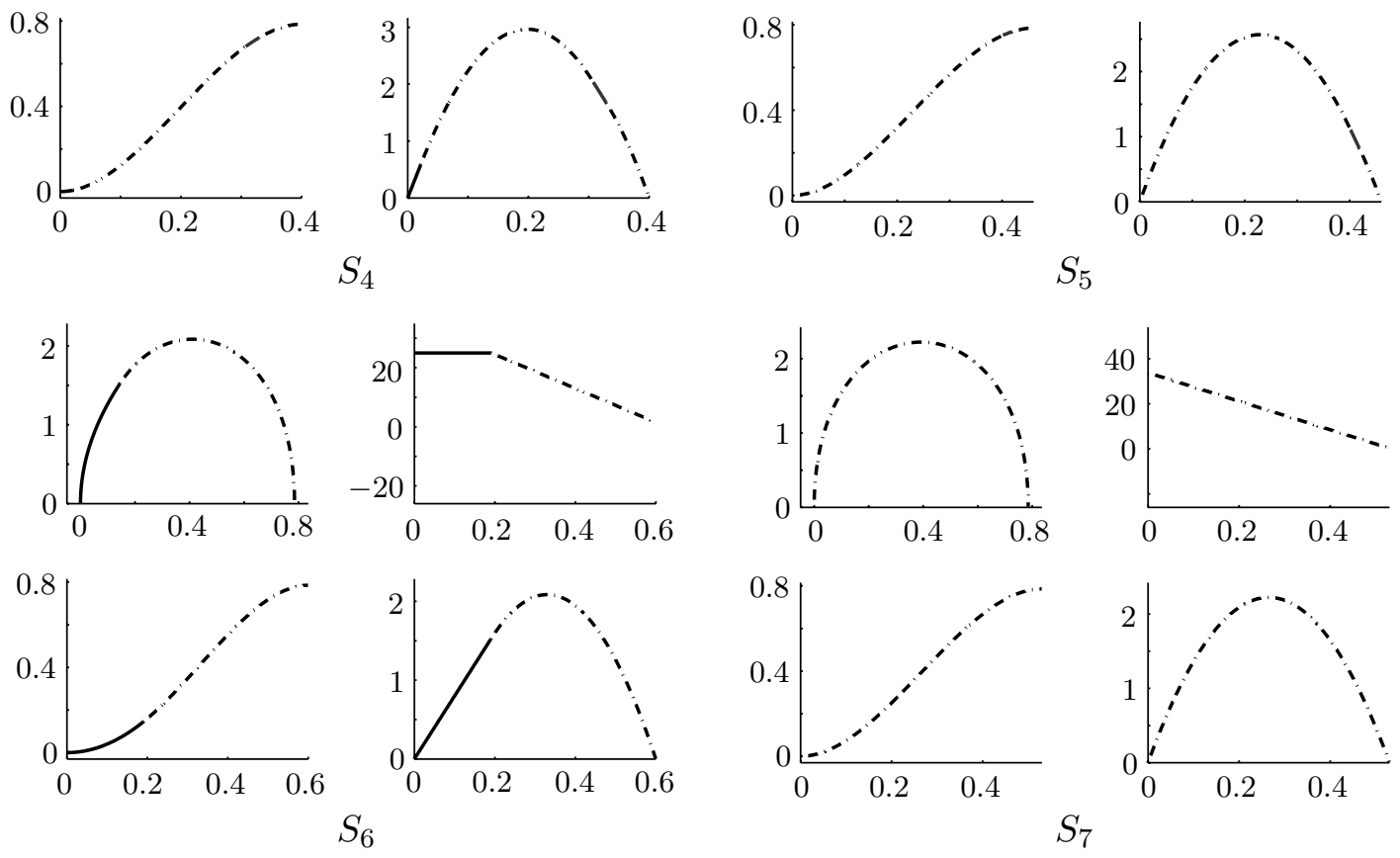

Fig. 3. Different optimal strategies.

Of course, we have $\tau_{i}>0$ for all $i$ and $\sum_{i=1}^{5} \tau_{i}=T$. This implies several constraints on $p_{0}$ and $q_{0}$. The initial adjoint vector can be computed by requiring that $y_{5}\left(\tau_{5}\right)=0$ and $x_{5}\left(\tau_{5}\right)=x_{\mathrm{t}}$. Explicit formulas for $p_{0}$ and $q_{0}$ cannot be obtained, but it is numerically easy to compute these values and to check if they are compatible with the above conditions.

The time $T_{1}$ after which this strategy is not actual can be easily determined numerically.

3.4. Cases $S_{3}$ and $S_{4}$. Bang only in $u_{\max }$ or only in $-u_{\min }$, four-piece trajectories. The disappearance of the saturation $u=u_{\max }$ is determined by $\tau_{1}=0$, which implies $q_{0}=q_{0}^{a}=$ $-2 k \alpha+2 \alpha u_{\max }$. 
Similarly, the disappearance of the saturation $u=-u_{\min }$ is determined by $\tau_{5}=0$, which implies

$$
q_{0}=q_{0}^{b}=-2 k \alpha+\frac{\alpha\left(u_{\max }^{2}+u_{\min }^{2}+2 k u_{\min }\right)}{u_{\max }-k} .
$$

It follows that

$$
q_{0}^{a} \geq q_{0}^{b} \quad \Leftrightarrow \quad u_{\max }-u_{\min } \geq 2 k .
$$

If $u_{\max }-u_{\min } \geq 2 k$, it is easy to see that we have to choose $q_{0}=q_{0}^{a}$ and $\tau_{1}=0$ before $\tau_{5}=0$.

Conversely, if $u_{\max }-u_{\min } \leq 2 k$, we should take $q_{0}=q_{0}^{b}$, and $\tau_{5}=0$ appears first.

We set $T=T_{1}^{a}$ in the first case and $T=T_{1}^{b}$ in the second case. We do not give more details for this case.

3.5. Case $S_{5}$. Three-piece trajectories. Note that this situation can appear only if $u_{\max } \geq 2 k$. Therefore, it may appear after each of the above situations (since we can have $u_{\max }-u_{\min } \leq 2 k$ or $u_{\max }-u_{\min } \geq 2 k$ ). When $T \in\left[T_{1}, T_{2}^{a}\right]$, with $T_{2}^{a}=\sqrt{6 \Delta_{x} / k}$, we get optimal solutions of the unconstrained problem.

3.6. Case $S_{6}$. Two-piece trajectories. It can be proven that this situation is only possible when $u_{\max } \leq 2 k$. Consequently, this situation occurs when $T \in\left[T_{1}^{b}, T_{2}^{b}\right]$, with $T_{2}^{b}=$ $\sqrt{6 \Delta_{x} /\left(u_{\max }-k\right)}$.

3.7. Case $S_{7}$. Singular piece only. Symmetric velocity profiles. This situation appears for $T \in\left[T_{2}^{a},+\infty\left[\right.\right.$ (if $u_{\max } \geq 2 k$ ) or $T \in\left[T_{2}^{b},+\infty\left[\right.\right.$ (if $u_{\max } \leq 2 k$ ).

For $t \in[0, T]$,

$$
\left\{\begin{array}{l}
u=\frac{q_{0}}{2 \alpha}+k+\frac{k-p_{0}}{2 \alpha} t \\
y=\frac{q_{0}}{2 \alpha} t+\frac{k-p_{0}}{4 \alpha} t^{2} \\
q=q_{0}+\left(\frac{q_{0}}{2 \alpha}+k-p_{0}\right) t+\frac{k-p_{0}}{4 \alpha} t^{2}, \\
x=x_{\mathrm{s}}+\frac{q_{0}}{4 \alpha} t^{2}+\frac{k-p_{0}}{12 \alpha} t^{3} .
\end{array}\right.
$$

It turns out that in this case velocity profiles are symmetric (the acceleration time equals the deceleration time).

3.8. Examples of each of the optimal trajectories. Figure 3 illustrates the different strategies, except the most usual strategy $S_{2}$, which was depicted in the introduction (see Fig. 2) with the addition of inertial control.

\section{SMOOTHING THE OPTIMAL STRATEGY}

As one can note in the practical experiments (moreover, this is clear whatever), the control has to be inertial; i.e., the control is absolutely continuous, with gradient bounds. Let us assume

$$
\left|\frac{d u}{d t}\right| \leq U
$$

This section is not very crucial for what we want to show. Since the results are intuitively clear, while their proofs are rather technical and long (use the maximum principle for inertial controls), we just state a few results and do not give the proofs.

We will focus on the situation $S_{2}$ (the most suitable in practice) and assume $\left(\mathcal{H}_{\mathrm{s}}\right)$ that $\left.T \in\right] T_{\mathrm{c}}, T_{1}[$ (open interval). 
Theorem 3. Under assumption $\left(\mathcal{H}_{\mathrm{s}}\right)$, optimal constrained solutions do exist, provided that the bound $U$ is large enough.

Theorem 4. When $U$ tends to infinity, under assumption $\left(\mathcal{H}_{\mathrm{s}}\right)$,

(1) optimal controls converge *-weakly to unconstrained optimal controls;

(2) constrained optimal state trajectories converge uniformly to unconstrained optimal state trajectories;

(3) optimal controls are seven pieces (counted here for upward motion): bounded maximum derivative, maximum bang, usual singular, inactivation, usual singular, minimum bang, and bounded maximum derivative.

An example of such a smoothed trajectory is depicted in Fig. 2 in the Introduction.

\section{THE INACTIVATION PRINCIPLE}

Let us consider the original system without the small-angle assumption, or even more generally any potential $\varphi(x)$ with constant sign.

Let us fix everything (the source, target, and the bounds) except the duration $T$ of the experiment. We also assume that $u_{\min }, u_{\max } \geq \sup _{x} \varphi^{\prime}(x)$.

Then, we still have a critical (minimum) time $T_{\mathrm{c}}$. Notice that for $T=T_{\mathrm{c}}$, the optimal control $u^{*}(t)$ changes sign: assuming the contrary, since $u^{*}(t)=-u_{\min }$ or $u_{\max }$ (minimum time and no abnormal trajectories), the motion would be monotonic and therefore cannot connect the source to the target.

An easy continuity argument shows that the same happens ( $u^{*}$ changes sign) in some time interval $] T_{\mathrm{c}}, T_{2}[$.

Lemma 1. Assume that $T \in] T_{\mathrm{c}}, T_{2}\left[\right.$. Then, the optimal control $u^{*}$ is continuous.

Proof. Along an optimal trajectory with $\lambda=-1$, since $T>T_{\mathrm{c}}$ is a nonminimum time $(\lambda$ is the adjoint additional variable), the Hamiltonian $H$ is

$$
H=-|y u|-\alpha(x, y) \dot{y}^{2}+p y+q\left(u-\varphi^{\prime}(x)\right) .
$$

Here we assume that $\alpha$ is strictly positive and continuous. In our case, along the optimal trajectories, $y$ does not change sign, and hence $\alpha(y)$ is continuous.

Therefore, $H(t)$ is a strictly concave Lipschitz continuous function of $u(t)$. It has to be maximum all along the trajectory, and the $u$ reaching the maximum is unique. The result follows.

Moreover, $u$ is uniquely determined by the inclusion

$$
0 \in \partial_{u} H,
$$

where $\partial_{u}$ is the subdifferential of convex analysis (with respect to $u$ ). This condition can be rewritten as

$$
u \in \frac{|y| \varepsilon+2 \alpha(x, y) \varphi^{\prime}(x)+q}{2 \alpha(x, y)}=I, \quad \text { where } \quad \varepsilon= \begin{cases}\operatorname{sign}(u) & \text { if } u \neq 0 \\ {[-1,1]} & \text { if } u=0\end{cases}
$$

Now, since $u$ is a continuous function of $t$, changing sign (for $y \neq 0$ ), $u$ has to cross the continuously time-varying interval $I$. Therefore, $u$ has to be zero on some time interval.

We have shown the following:

Theorem 5 (total inactivation principle). Under the assumptions of this section (in particular, in the case of our system without the small-angle assumption), for $T \in] T_{\mathrm{c}}, T_{2}[$, there is a nondegenerate subinterval of $[0, T]$ on which $u=0$ (total inactivation). 
The time $T_{2}$ at which total inactivation disappears is of importance for neurobiologists. We have computed it under the small-angle assumption:

$$
\begin{array}{ll}
T_{2}=\sqrt{\frac{6 \Delta_{x}}{u_{\max }-k}} & \text { for } u_{\max } \leq 2 k, \\
T_{2}=\sqrt{\frac{6 \Delta_{x}}{k}} & \text { elsewhere. }
\end{array}
$$

\section{IDEAS FOR THE MAIN COMPUTATIONS}

Computations are tedious but easy: optimal control of a linear system with strictly convex (piecewise quadratic) cost. Hence, all the results of Section 3 are obtained just playing with the maximum principle.

Moreover, it can be shown (by comparisons) that the optimal trajectories are entirely in $\{y \geq 0\}$ or $\{y \leq 0\}$. Therefore, there is just nonsmoothness with respect to $u$, and we need only the usual Pontryagin's maximum principle.

The Hamiltonian $\mathcal{H}$ of the problem can be written as

$$
\mathcal{H}=-\lambda\left(y|u|+\alpha(u-k)^{2}\right)+p y+q(u-k),
$$

where $\lambda \geq 0$ is the constant additional adjoint variable and $(p, q)$ is the adjoint vector to $(x, y)$.

We set $z=q-y$ and $w=q+y$. The condition $y \geq 0$ is now $w \geq z$.

Let us just show the $(z, w)$ phase portrait of the optimal trajectories, in a single case (the one corresponding to the most usual situation, $p_{0}>k$, see Fig. 4).

The typical trajectory drawn in the half-plane $y \geq 0(w \geq z)$ corresponds to the most usual trajectories $S_{2}$.

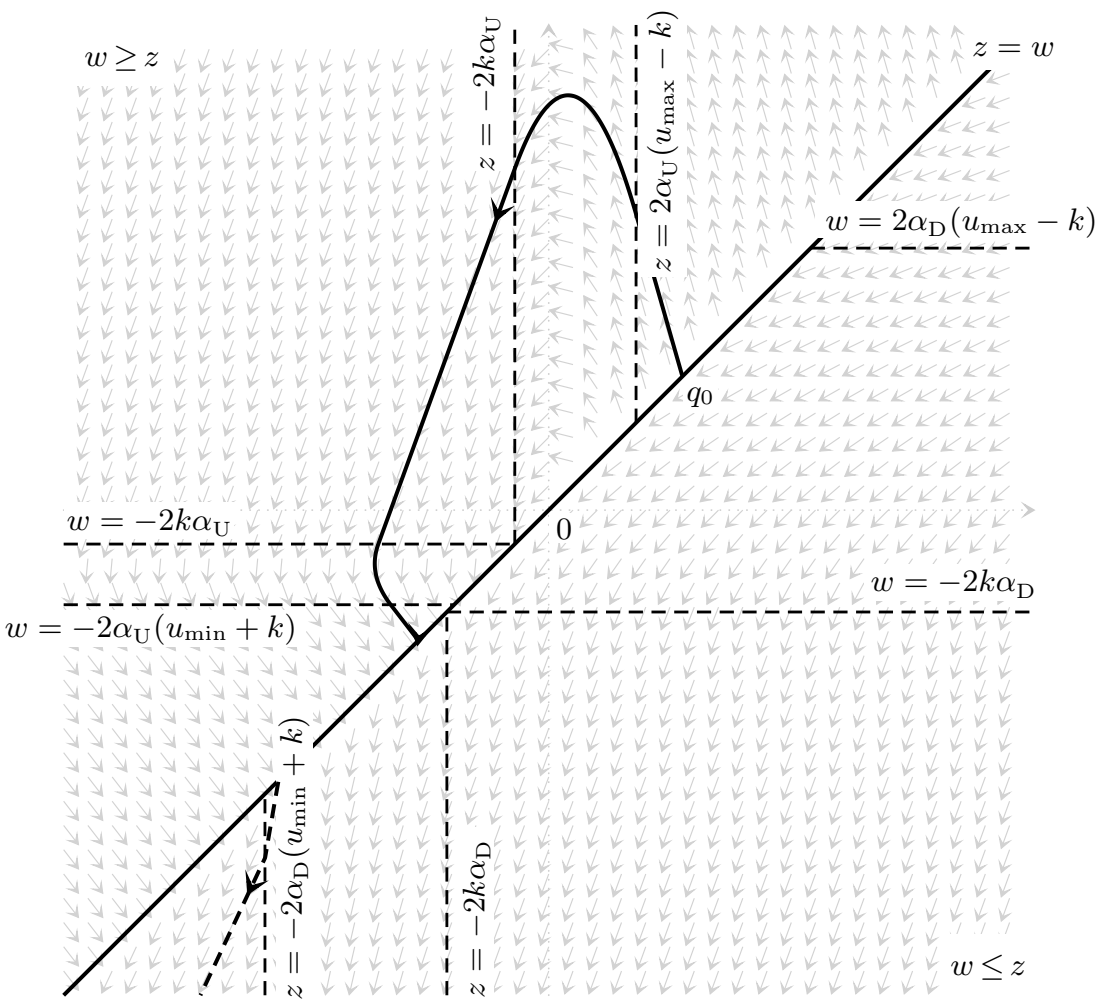

Fig. 4. Phase portrait for $p_{0}>k$. 


\section{ACKNOWLEDGMENTS}

This work was partly supported by the "Centre National d'Etudes Spatiales" and the "Conseil Regional de Bourgogne."

\section{REFERENCES}

1. L. S. Pontryagin, V. G. Boltyanskii, R. V. Gamkrelidze, and E. F. Mishchenko, The Mathematical Theory of Optimal Processes (Fizmatgiz, Moscow, 1961; Pergamon Press, Oxford, 1964).

2. E. B. Lee and L. Markus, Foundations of Optimal Control Theory (J. Wiley and Sons, New York, 1967).

3. J.-P. Gauthier and I. Kupka, Deterministic Observation Theory and Applications (Cambridge Univ. Press, Cambridge, 2001)

4. E. Todorov, "Optimality Principles in Sensorimotor Control," Nat. Neurosci. 7 (9), 907-915 (2004).

5. C. Papaxanthis, T. Pozzo, and M. Schieppati, "Trajectories of Arm Pointing Movements on the Sagittal Plane Vary with Both Direction and Speed," Exp. Brain Res. 148 (4), 498-503 (2003).

6. R. Gentili, V. Cahouet, and C. Papaxanthis, "Motor Planning of Arm Movements Is Direction-Dependent in the Gravity Field," Neuroscience 145 (1), 20-32 (2007).

This article was submitted by the authors in English 